\title{
A marginal liver graft with hyperbilirubinemia transplanted successfully by ischemia-free liver transplantation
}

\author{
Changjun Huang ${ }^{1,2,3 \#}$, Zhitao Chen ${ }^{1,2,3 \#}$, Tielong Wang ${ }^{1,2,3}$, Xiaoshun $\mathrm{He}^{1,2,3}$, Maogen Chen ${ }^{1,2,3}$, \\ Weiqiang $\mathrm{Ju}^{1,2,3}$
}

${ }^{1}$ Organ Transplant Center, The First Affiliated Hospital, Sun Yat-sen University, Guangzhou, China; ${ }^{2}$ Guangdong Provincial Key Laboratory of Organ Donation and Transplant Immunology, Guangzhou, China; ${ }^{3}$ Guangdong Provincial International Cooperation Base of Science and Technology (Organ Transplantation), Guangzhou, China

\#These authors contributed equally to this work.

Correspondence to: Xiaoshun He; Maogen Chen; Weiqiang Ju. Organ Transplant Center, The First Affiliated Hospital, Sun Yat-sen University, No. 58 Zhongshan Er Road, Guangzhou 510080, China. Email: hexsh@mail.sysu.edu.cn; chenmg3@mail.sysu.edu.cn; weiqiangju@163.com.

\begin{abstract}
The shortage of transplant organs remains a serious issue worldwide, and using liver grafts from extended criteria donors could expand the donor pool. Extended criteria donor liver allografts have a high chance of complications such as primary nonfunction, early allograft dysfunction, and ischemictype biliary lesions. How to employ these extended criteria donors safely and effectively warrants further investigation. Herein, we report the successful use of a marginal donor liver with hyperbilirubinemia to save the life of an acute-on-chronic liver failure recipient using a new surgical technique: ischemia-free liver transplantation (IFLT). The graft was retrieved for transplantation due to the following reasons: (I) the recipient was in a life-threatening situation and no living donor donation candidate was available; (II) the graft was considered transplantable except for cholestasis; and (III) IFLT could reduce ischemia/reperfusion injury (IRI), resuscitate the allograft $e x s i t u$, and maintain organ viability before transplantation. The graft was transplanted successfully using the IFLT procedure. Although anatomic biliary stricture occurred after surgery, no IRI-related complications were found during the follow-up. The use of liver grafts from extended criteria donors is safe and effective under IFLT. Additional IFLT clinical studies need to be performed, particularly concerning donor management, graft selection, and ex situ resuscitation.
\end{abstract}

Keywords: Ischemia-free liver transplantation (IFLT); hyperbilirubinemia; extended criteria donors; liver failure; case report

Submitted Sep 06, 2020. Accepted for publication Jan 12, 2021.

doi: $10.21037 /$ atm-20-6296

View this article at: http://dx.doi.org/10.21037/atm-20-6296

\section{Introduction}

Organ transplantation is a critical life-saving therapy for end-stage organ failure (1). According to data from the World Health Organization (WHO) Global Observatory on Donation and Transplantation (http://www.transplantobservatory.org/), organ shortage is a major limitation for transplantation, and solid organ transplants account for fewer than $10 \%$ of the global demand. It is thus crucial to expand the organ donor pool, and various approaches have been attempted, including expanding the organ source pool to deceased donor organ donation, living-related organ donation, split or reduced-size organ transplantation, and the use of extended criteria donors (ECDs) or marginal donors $(2,3)$. Because of the increased mortality of patients on the waiting list, a growing number of ECD grafts are used in liver transplantation (LTx) (4). In human LTx, ischemia/reperfusion injury (IRI) occurring following blood flow restoration can cause damage to the grafts, leading to complications such as early allograft dysfunction (EAD), primary nonfunction (PNF), and ischemic-type biliary 
lesions (ITBLs) (5). IRI can also cause damage to other organs, leading to complications such as postreperfusion syndrome (PRS) and acute kidney injury (AKI) (6). The tolerance to hypoxia and IRI in ECD or marginal donor livers is worse than that of high-quality allografts, leading to a higher incidence of IRI-related complications $(7,8)$. To enhance the quality of ECD or marginal allografts, machine perfusion, including hypothermic machine perfusion, normothermic machine perfusion (NMP), and subnormothermic machine perfusion, has been demonstrated to be a viable preservation strategy in LTx (9). The first human LTx with NMP-preserved grafts was reported by Ravikumar et al. in 2016, and their results confirmed the safety and feasibility of using NMP in organ transplantation and transportation (10). Subsequently, a clinical randomized controlled trial demonstrated that NMP preservation is associated with a $50 \%$ lower level of graft injury and a 50\% lower organ discard rate than conventional static cold storage (11). Mechanistic studies have revealed that NMP not only inhibits inflammatory reactions but also promotes graft regeneration in the donor liver, leading to a decreased IRI level (12). Based on the NMP technique and surgical innovation, ischemiafree liver transplantation (IFLT) was established at our institute, theoretically eliminating the effects of donor liver IRI throughout the transplant process. The first case of IFLT involved the successful resuscitation of a donor liver with $85-90 \%$ macrovesicular steatosis (13), suggesting its considerable potential in expanding the organ donor pool.

Herein, we report the successful application of a hyperbilirubinemia graft using IFLT and discuss the issues concerning the use of grafts from ECDs, donor management, and the timing of transplantation.

We present the following case in accordance with the CARE reporting checklist (available at http://dx.doi. org/10.21037/atm-20-6296).

\section{Case presentation}

A 35-year-old man was diagnosed with chronic viral hepatitis B and was taking entecavir irregularly for 6 years. He had no history of smoking, alcohol consumption, or other types of viral hepatitis. He had been admitted to a local hospital and started to present with symptoms related to acute-on-chronic liver failure (ACLF) for 3 weeks, including jaundice, severe coagulation dysfunction, and refractory ascites. After this, the patient's condition worsened gradually and he was referred to our institute as an LTx candidate. While waiting for a suitable donor liver, he eventually developed gastrointestinal bleeding and grade 4 hepatic encephalopathy before transplantation. His laboratory tests showed severe liver function decompensation [aspartate aminotransferase (AST), $40 \mathrm{U} / \mathrm{L}$; alanine transaminase (ALT), $69 \mathrm{U} / \mathrm{L} ; \gamma$-glutamyltransferase (GGT), $70 \mathrm{U} / \mathrm{L}$; total bilirubin (TBIL), $38.2 \mathrm{mg} / \mathrm{dL}$; direct bilirubin (DBIL), $21.2 \mathrm{mg} / \mathrm{dL}$; international normalized ratio (INR), 3.68; activated partial thromboplastin time, $73.5 \mathrm{~s}$; fibrinogen, $0.85 \mathrm{~g} / \mathrm{L}$; prothrombin activity, $18 \%$; hemoglobin, $56 \mathrm{~g} / \mathrm{L}$; platelet, $60 \times 10^{9} / \mathrm{L}$; white blood cell, $2.62 \times 10^{9} / \mathrm{L}$; blood ammonia, $\left.108 \mu \mathrm{mol} / \mathrm{L}\right]$, and his model for end-stage liver disease (MELD) score was 32. In addition to conventional anti-hepatitis $B$ virus treatment and measures to protect hepatic function, he had undergone plasma exchanges or dialysis treatment 4 times to reduce the toxicity of hyperbilirubinemia and mitigate inflammatory mediators before LTx. The treatment effect was not satisfactory, and LTx was the only promising treatment approach that could provide freedom from life-threatening conditions.

A brain-dead man aged 26 years voluntarily donated his organs. The cause of brain death was severe traumatic brain injury. He had undergone hematoma evacuation and decompressive craniectomy surgery immediately after admission to the local hospital. He remained in a deep coma, with the disappearance of spontaneous respiration and absence of a brainstem reflex after surgery. The declaration of brain death was made by 2 independent qualified neurologists and critical care physicians. The laboratory tests revealed pulmonary infection, hypernatremia, and liver dysfunction. In particular, his serum TBIL level had gradually increased to $14.03 \mathrm{mg} / \mathrm{dL}$. When he was transferred to our hospital, he had been admitted to the local hospital for 25 days. After being transferred to our hospital, enhanced anti-infection and sodium-lowering therapies were initiated, magnesium isoglycyrrhizinate and ursodeoxycholic acid were used to improve liver function, intravenous nutrition was reduced, and enteral nutrition was gradually increased. The indocyanine green retention rate at 15 minutes (ICG15) 7 days before retrieval was $42.5 \%$, and at 1 day before retrieval the rate was $27.5 \%$. The laboratory data before donor organ retrieval are summarized in Table 1, and indicated that his liver function was gradually improving, the retrieval should be delayed, and that donor management should be continued. However, 
Table 1 Blood biochemical parameters of the 26-year-old brain-dead donor.

\begin{tabular}{|c|c|c|c|c|c|c|c|c|c|c|}
\hline Parameters & \multicolumn{9}{|c|}{ Days before donor retrieval } & $\begin{array}{l}\text { Day of } \\
\text { retrieva }\end{array}$ \\
\hline ALT, U/L & 128 & 107 & 125 & 158 & 203 & 212 & 180 & 144 & 100 & 84 \\
\hline AST, U/L & 227 & 135 & 216 & 410 & 437 & 315 & 183 & 88 & 75 & 61 \\
\hline TBIL, mg/dL & 1.11 & 9.85 & 13.71 & 14.03 & 13.46 & 14.03 & 9.64 & 6.05 & 5.46 & 4.88 \\
\hline GGT, U/L & 37 & 51 & 52 & 24 & 21 & 26 & 27 & 26 & 28 & 30 \\
\hline $\mathrm{PA}, \mathrm{mg} / \mathrm{L}$ & NA & NA & 63 & 78 & 113 & 156 & 185 & 186 & 186 & 185 \\
\hline $\mathrm{Na}^{+}, \mathrm{mmol} / \mathrm{L}$ & 158 & 175 & 160 & 156 & 152 & 151 & 151 & 148 & 136 & 133 \\
\hline
\end{tabular}

ALT, alanine transaminase; AST, aspartate aminotransferase; DBIL, direct bilirubin; GGT, $\gamma$-glutamyltransferase; PA, prealbumin; Na ${ }^{+}$, sodium ion; NA, not applicable; TBIL, total bilirubin.

because the condition of the recipient required urgent attention and the donor was young without underlying liver disease, we decided to retrieve the liver from the donor in advance using the IFLT procedure.

Our patient successfully underwent LTx via the IFLT procedure. The liver of the donor was dark brown (Figure 1A), and no hepatic artery variant was documented in the computed tomography angiography examination. The pathological findings of a liver biopsy specimen revealed mild cholestasis and hydropic degeneration, without other abnormal findings (Figure $1 B$ ). The liver graft had undergone NMP for 440 minutes until allograft revascularization. The perfusate components are reported in our previous article (13). The $\mathrm{pH}$ value of the perfusate was in the range of 7.16 to 7.30 at the initial stage and maintained at a normal range (7.35-7.45) after 100 minutes. The lactate levels dropped quickly from $8.3 \mathrm{mmol} / \mathrm{L}$ to $0.6 \mathrm{mmol} / \mathrm{L}$ within 40 minutes and gradually stabilized at approximately $2.0 \mathrm{mmol} / \mathrm{L}$ (Figure $1 C$ ). Bile production was continuous, and the total volume was approximately $23 \mathrm{~mL}$ (Figure 1D). The $\mathrm{pH}$ values of the bile were higher than detected $(>7.8)$. The flow and pressure of both the portal vein and hepatic artery were acceptable throughout the entire IFLT procedure (Figure $1 E$ and $F$ ). The partial pressure of oxygen $\left(\mathrm{pO}_{2}\right)$ was stabilized at approximately $250 \mathrm{mmHg}$, and the partial pressure of carbon dioxide $\left(\mathrm{pCO}_{2}\right)$ was stabilized at approximately $35 \mathrm{mmHg}$ during the preservation phase (Figure 1G). The TBIL, DBIL, ALT, and AST in the perfusate gradually increased and were acceptable at the end of perfusion $(4.19 \mathrm{mg} / \mathrm{dL}, 3.29 \mathrm{mg} / \mathrm{dL}$, $477 \mathrm{U} / \mathrm{L}$, and 1,154 U/L, respectively; Figure $1 H$ and $I$ ). Taken together, these results suggested efficient NMP and transplantable organ viability. The cold ischemic time and warm ischemic time were both 0 minutes. Cavo-caval anastomosis was performed using a piggy-back technique. Reconstruction of the portal vein and hepatic artery were performed in an end-to-end fashion. The duration of LTx was 8 hours 45 minutes, and the anhepatic phase was 68 minutes. The estimated blood loss was approximately $5,500 \mathrm{~mL}$.

The conventional immunosuppressive regimen in our unit comprises basiliximab, calcineurin inhibitors, and mycophenolate mofetil. Hormones are not routinely used in our center. However, because of the slow decline of bilirubin in the early postoperative period, we used a small dose of methylprednisolone. After methylprednisolone use, bilirubin gradually decreased. The donor's CYP3A5*3 (A6986G) genetic locus was AG, and the recipient's corresponding locus was AA. Tacrolimus was first administered on postoperative day (POD) 4 but a therapeutic level was difficult to attain because of a rapid metabolic pattern. Cyclosporine A was used on POD 14, and the concentration was satisfactory. Rapid recovery of blood coagulation function and liver enzymes after surgery was recorded. His graft function continued to improve, and he was discharged with good graft function on POD 22. Unfortunately, he required a stent to treat anastomotic stricture of the common bile duct using endoscopic retrograde cholangiopancreatography on POD 44, and the stent was removed on POD 321. No PRS, AKI, ITBLs, or rejection occurred after surgery. Figure 2 shows a summary of his clinical course. At follow-up, the recipient showed good allograft function and has enjoyed a good quality of life for more than 1 year and as of the publication of this 
A

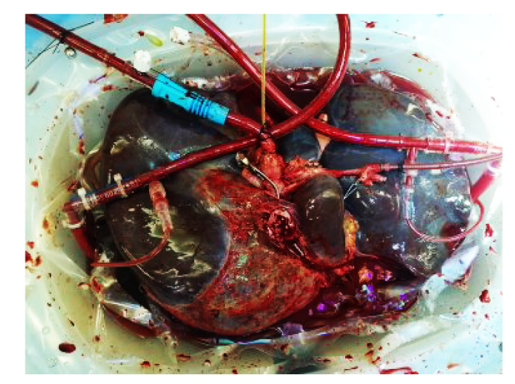

D

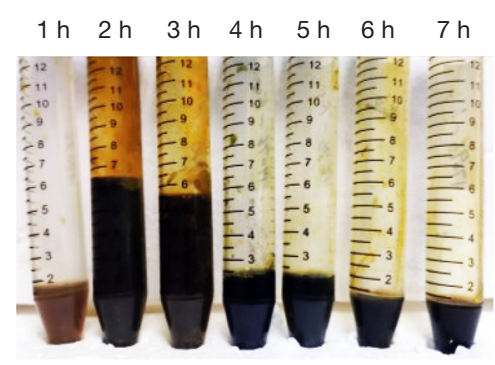

G

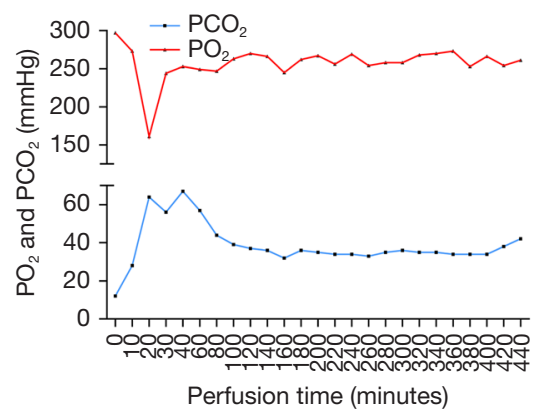

B

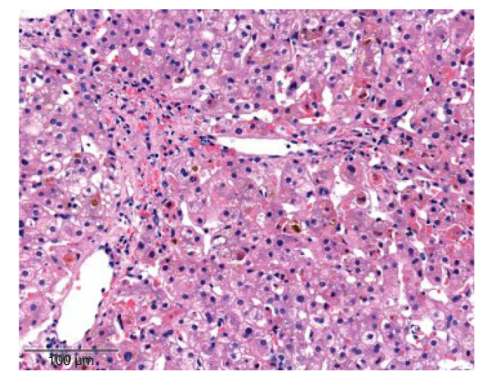

E

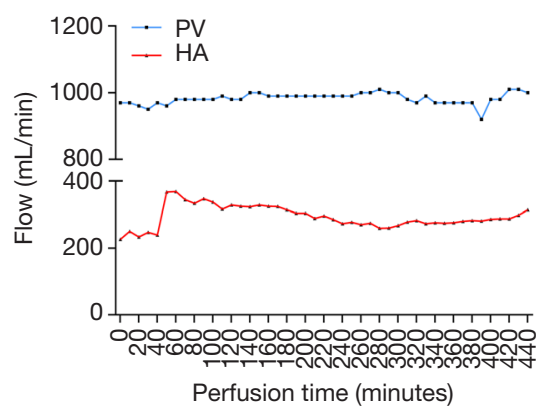

$\mathrm{H}$

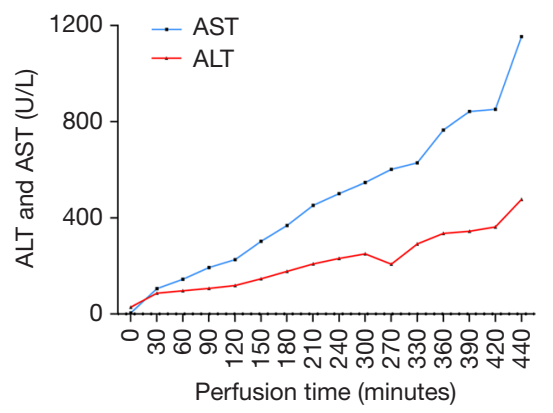

C

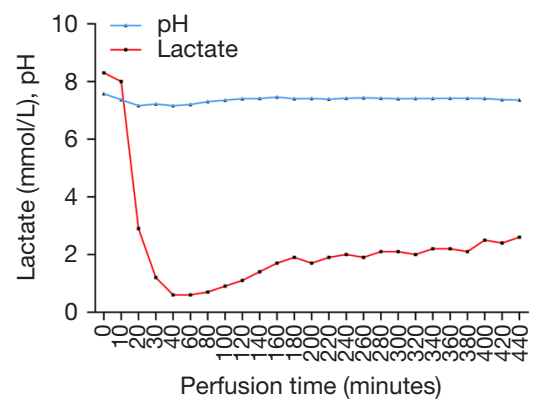

F

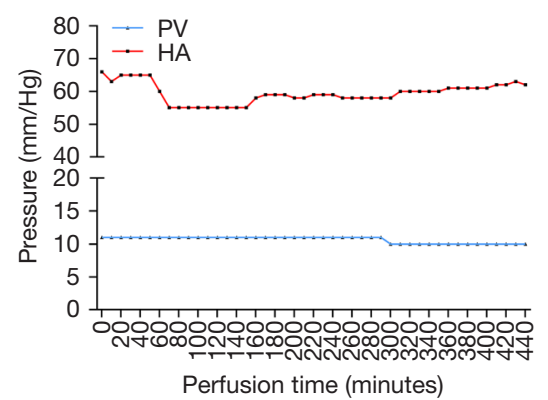

I

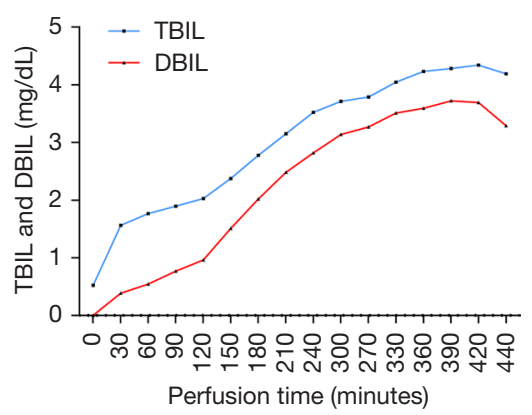

Figure 1 Histopathological and normothermic machine perfusion characteristics of the donor liver. (A) Shows the liver appearance under ex situ perfusion; (B) shows histopathological features (hematoxylin-eosin stain, 200x); (C) shows $\mathrm{pH}$ values and lactate levels in the perfusate; (D) shows bile production; (E and F) show the flow rates and pressure of the hepatic artery (HA)/portal vein (PV); (G, H, and I) show the $\mathrm{O}_{2}$ and $\mathrm{CO}_{2}$ tension and liver function tests [alanine transaminase (ALT), aspartate aminotransferase (AST); total bilirubin (TBIL), direct bilirubin (DBIL)] in the perfusate.

paper.

All the procedures in the studies involving human participants were performed in accordance with the Helsinki Declaration (as revised in 2013), and this study was approved by the ethics committee of The First Affiliated Hospital, Sun Yat-sen University \{no. [2019]037\}. Written informed consent was obtained from the patient for the publication of this article. The organ used in this study was procured from a brain-dead volunteer donor with written informed consent from all the directive family members.

\section{Discussion}

ECD allografts are gradually being accepted for transplantation because of the increasing demand for donor organs. Despite the beneficial effect on reducing wait times, the use of ECD allografts has also been associated with lower post-transplant outcomes caused by their susceptibility to IRI and subsequent decreased functional recovery (14). Although no unified criteria exists for the definition of ECD or marginal livers, steatosis, age, serum sodium, the ICU stay time, and serum bilirubin are frequently adopted 

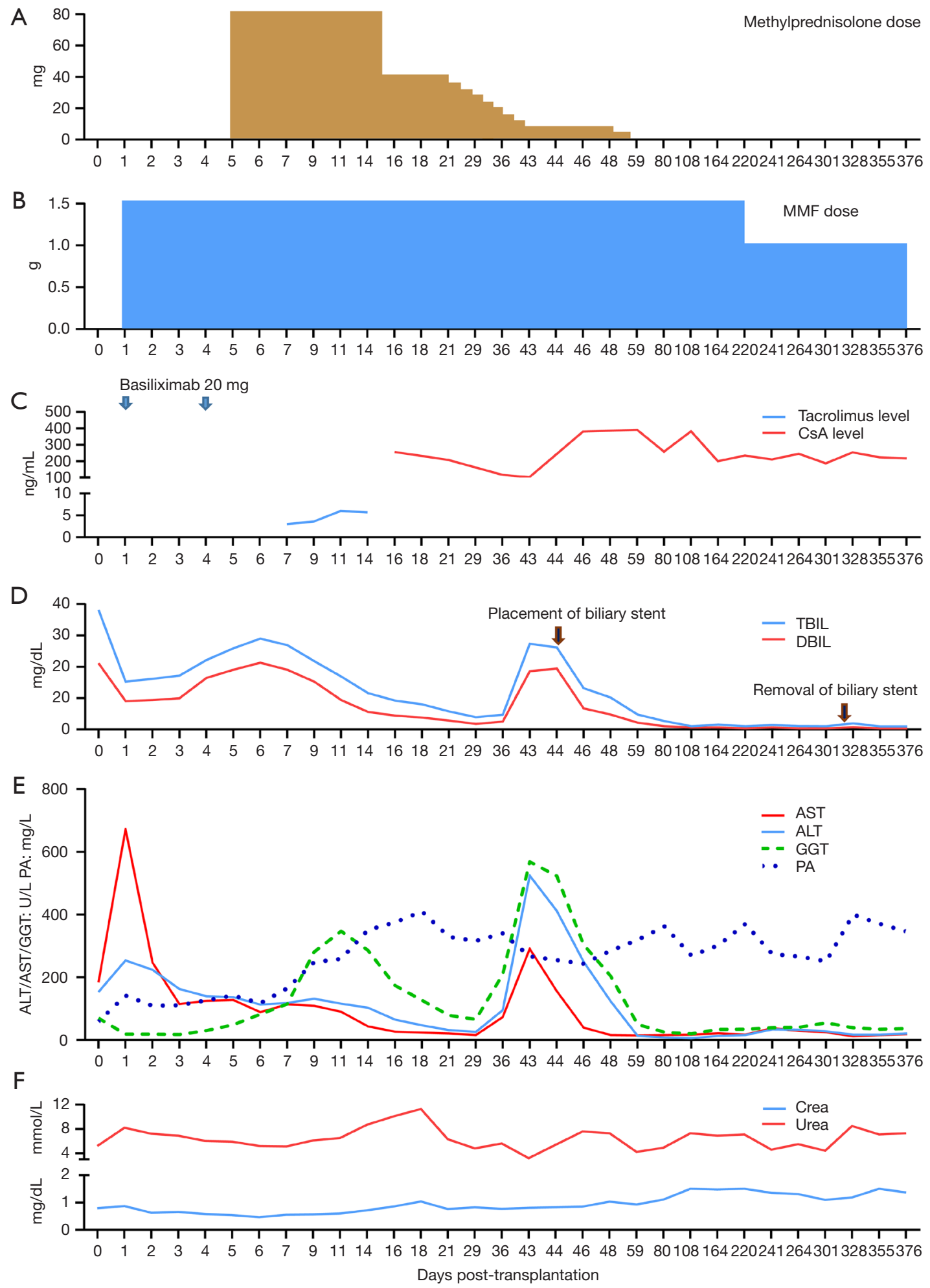

Figure 2 Posttransplant course of the recipient who received a hyperbilirubinemia donor liver during follow-up. (A, B, C, and D) show the methylprednisolone, mycophenolate mofetil (MMF), basiliximab dose, and tacrolimus/cyclosporine A (CsA) levels. (D, E, and F) show the changes in the total bilirubin (TBIL), direct bilirubin (DBIL), alanine transaminase (ALT), aspartate aminotransferase (AST), $\gamma$-glutamyl transpeptidase (GGT), prealbumin (PA), creatinine (Crea), and urea. 
parameters to evaluate the organ utilization risk at the various transplant centers (15). According to the latest Eurotransplant manual (version 5.0 January 30, 2017, https://www.eurotransplant.org/patients/eurotransplantmanual/), donors with a serum bilirubin level $>3.0 \mathrm{mg} / \mathrm{dL}$ are considered to be "marginal donors". In more extreme situations, Czerwiński et al. reported that livers with a bilirubin level $>2 \mathrm{mg} / \mathrm{dL}$ from alcohol abuse donors might not be suitable for transplantation because of the high incidence of severe histological changes (16). The donor in our case had a high serum bilirubin level of $4.88 \mathrm{mg} / \mathrm{dL}$ on the retrieval day, with a peak serum bilirubin level of $14.03 \mathrm{mg} / \mathrm{dL}$. The ICG clearance test is the most common and convenient approach for the perioperative dynamic evaluation of liver function in the case of hepatectomy and LTx (17). The donor's ICG15 values in our case at -7 days and -1 day before retrieval were far beyond the normal value $(<10 \%)$. The ICG clearance values may be misguided in the case of hyperbilirubinemia because of the carrier competition (18). Hyperbilirubinemia in our case limited the predictive value of the ICG clearance test. However, the relatively high level of ICG15 still reflected that the donor liver was not in good condition. According to our experience, the donor liver was not suitable at that time for conventional LTx and needed continued maintenance.

We ultimately retrieved and accepted the donation in advance for the following reasons. First, the recipient was diagnosed with ACLF and presented with hepatic coma, severe coagulopathy, and liver disorders, with an urgent need for transplantation. According to previously described diagnostic criteria of ACLF grades, the patient had grade 3 ACLF (liver: serum bilirubin $>12 \mathrm{mg} / \mathrm{dL}$; brain: grade IV hepatic encephalopathy; coagulation: INR $>2.5$ ). Gustot et al. reported that nonremission grade 3 ACLF patients have a very high 28-day transplant-free mortality (96.6\%) (19), but no appropriate living donor donation among the family members was found. Over time, he might have lost the chance of transplantation because of the increasing risk of infection, or brain, kidney, and other organ dysfunction. Second, the donor was young without underlying liver disease, and a liver biopsy of the graft showed no abnormal findings other than mild cholestasis and hydropic degeneration. Third, because of the IFLT technique, we could resuscitate the donor liver and assess liver viability ex situ under continuous NMP.

NMP has been successfully used to resuscitate ECD or marginal livers, prolong the liver preservation time, and reduce IRI-related complications $(11,20,21)$. The cold ischemia time can be reduced using NMP, while graft ischemia still cannot be fully avoided during the conventional procedure of organ procurement, preparation, and implantation (22). We hypothesized that LTx without graft ischemia might achieve better outcomes. We set up a reliable IFLT procedure after a series of animal experiments supported by NMP and successfully applied it to humans in 2017 (13). During IFLT, the oxygenated blood supply was persistent throughout the whole process of procurement, preservation, and implantation. According to our previous pilot study, the incidence of EAD and the peak levels of liver damage markers significantly declined with the use of IFLT (23). The IFLT procedure has the following advantages: (I) complete avoidance of graft ischemia and IRI, (II) assessment of graft viability before transplantation, and (III) the potential to resuscitate unusable grafts ex situ, ameliorating graft damage and enabling subsequent transplantation. Livers were considered transplantable if they met at least 2 of the following criteria during ex situ NMP (24): (I) metabolized lactate $\leq 2.5 \mathrm{mmol} / \mathrm{L}$ within 4 hours from the start of the perfusion; (II) bile production; (III) a $\mathrm{pH}$ greater than 7.30 in the perfusate; (IV) stable hepatic artery flow $(\geq 150 \mathrm{~mL} / \mathrm{min})$ and portal vein flow ( $\geq 500 \mathrm{~mL} / \mathrm{min})$; (V) homogeneous graft perfusion with a soft consistency of the parenchyma. Although the graft in this case appeared dark brown and biopsy revealed cholestasis, the perfusion parameters met all the above criteria during NMP. The peak AST and ALT within 7 days post-transplantation were 672 and $254 \mathrm{U} / \mathrm{L}$, respectively, and the INR on POD 7 was 1.22 . However, the posttransplant bilirubin level decreased more slowly than expected, and the initial dose of $80 \mathrm{mg}$ of methylprednisolone was used to improve liver function according to our previous randomized controlled trial (25). No IRI-related IBTLs were found after follow-up up to 376 days postoperation. The efficacy and safety of IFLT require more in-depth study, and a randomized controlled trial (ChiCTR1900021158) is ongoing in our center.

Currently, more than $80 \%$ of organ donations in our center come from brain-dead donors. Nonetheless, brain death is often accompanied by dramatic pathophysiological changes, which, without proper management, can result in the degeneration of organ function before retrieval (26). Brain-dead patients require aggressive and intensive care from the declaration of brain death until organ retrieval, including sustaining normal temperature, infection control, management of the respiratory/circulatory system, and replacement of hormones (27). A previous study showed that 
the incidence of early graft loss in donors with a final serum sodium level $>155 \mathrm{mmol} / \mathrm{L}$ to be significantly elevated, with early graft survival in liver donors with corrected hypernatremia being unaffected (28). Hypernatremia was present in this donor; however, by restriction of the sodium supply, utilization of vasopressin, and nasal feeding with sterile injection water, the serum sodium was successfully reduced to $133 \mathrm{mmol} / \mathrm{L}$. We speculated that the cause of hyperbilirubinemia might have been due to infection and long-term intravenous nutrition at the local hospital. After enhanced anti-infection and enteral nutrition treatment, the donor's bilirubin dropped gradually. Although the bilirubin level fluctuated before retrieval, we speculated that if the maintenance was continued, the bilirubin should continue to decrease. However, organ retrieval using the IFLT technique is also safe and effective under some circumstances, particularly when the recipient is in a lifethreatening situation or vital signs of the donor are difficult to maintain.

In conclusion, we presented a case of successful LTx using a hyperbilirubinemia liver graft with an IFLT procedure. Particular attention should be paid to the use of ECD livers and achieving a balance between whether the donor organ is retrieved or continually maintained.

\section{Acknowledgments}

Funding: This work was supported by the National Natural Science Foundation of China (No. 81401324 and 81770410), the Guangdong Basic and Applied Basic Research Foundation (No. 2020A1515011557 and 2020A1515010903), the Guangdong Provincial Key Laboratory of Organ Donation and Transplant Immunology (No. 2013A061401007 and 2017B030314018), and the Guangdong Provincial International Cooperation Base of Science and Technology (Organ Transplantation) (No. 2015B050501002).

\section{Footnote}

Reporting Checklist: The authors have completed the CARE reporting checklist. Available at http://dx.doi.org/10.21037/ atm-20-6296

Conflicts of Interest: All authors have completed the ICMJE uniform disclosure form available at http://dx.doi. org/10.21037/atm-20-6296). The authors have no conflicts of interest to declare.
Ethical Statement: The authors are accountable for all aspects of the work in ensuring that questions related to the accuracy or integrity of any part of the work are appropriately investigated and resolved. All procedures performed in studies involving human participants were in accordance with the Helsinki Declaration (as revised in 2013), and this study was approved by the ethics committee of The First Affiliated Hospital, Sun Yat-sen University \{no. [2019]037\}. Written informed consent was obtained from the patient for the publication of this article. The organ used in this study was procured from a brain-dead volunteer donor with written informed consent from all the directive family members.

Open Access Statement: This is an Open Access article distributed in accordance with the Creative Commons Attribution-NonCommercial-NoDerivs 4.0 International License (CC BY-NC-ND 4.0), which permits the noncommercial replication and distribution of the article with the strict proviso that no changes or edits are made and the original work is properly cited (including links to both the formal publication through the relevant DOI and the license). See: https://creativecommons.org/licenses/by-nc-nd/4.0/.

\section{References}

1. Saidi RF, Hejazii Kenari SK. Challenges of organ shortage for transplantation: solutions and opportunities. Int J Organ Transplant Med 2014;5:87-96.

2. Wall SP, Plunkett C, Caplan A. A Potential Solution to the Shortage of Solid Organs for Transplantation. JAMA 2015;313:2321-2.

3. Witjes M, Kotsopoulos AMM, Otterspoor L, et al. The Implementation of a Multidisciplinary Approach for Potential Organ Donors in the Emergency Department. Transplantation 2019;103:2359-65.

4. Vodkin I, Kuo A. Extended Criteria Donors in Liver Transplantation. Clin Liver Dis 2017;21:289-301.

5. Verhoeven CJ, Farid WR, de Jonge J, et al. Biomarkers to assess graft quality during conventional and machine preservation in liver transplantation. J Hepatol 2014;61:672-84.

6. Tokodai K, Lannsjo C, Kjaernet F, et al. Association of post-reperfusion syndrome and ischemia-reperfusion injury with acute kidney injury after liver transplantation. Acta Anaesthesiol Scand 2020;64:742-50.

7. Sakamoto S, Sasaki K, Uchida H, et al. A severely cholestatic liver graft can be successfully used in deceased 
donor liver transplantation. Hepatol Res 2018;48:E367-71.

8. Situmorang GR, Sheerin NS. Ischaemia reperfusion injury: mechanisms of progression to chronic graft dysfunction. Pediatr Nephrol 2019;34:951-63.

9. Graham JA, Guarrera JV. "Resuscitation" of marginal liver allografts for transplantation with machine perfusion technology. J Hepatol 2014;61:418-31.

10. Ravikumar R, Jassem W, Mergental H, et al. Liver Transplantation After Ex Vivo Normothermic Machine Preservation: A Phase 1 (First-in-Man) Clinical Trial. Am J Transplant 2016;16:1779-87.

11. Nasralla D, Coussios CC, Mergental H, et al. A randomized trial of normothermic preservation in liver transplantation. Nature 2018;557:50-6.

12. Jassem W, Xystrakis E, Ghnewa YG, et al. Normothermic Machine Perfusion (NMP) Inhibits Proinflammatory Responses in the Liver and Promotes Regeneration. Hepatology 2019;70:682-95.

13. He X, Guo Z, Zhao Q, et al. The first case of ischemiafree organ transplantation in humans: A proof of concept. Am J Transplant 2018;18:737-44.

14. Czigany Z, Lurje I, Schmelzle M, et al. IschemiaReperfusion Injury in Marginal Liver Grafts and the Role of Hypothermic Machine Perfusion: Molecular Mechanisms and Clinical Implications. J Clin Med 2020;9:846.

15. Bruzzone P, Giannarelli D, Adam R, et al. A preliminary European Liver and Intestine Transplant AssociationEuropean Liver Transplant Registry study on informed recipient consent and extended criteria liver donation. Transplant Proc 2013;45:2613-5.

16. Czerwinski J, Perkowska A, Mroz A, et al. Assessment of cadaveric livers discarded from transplantation. A correlation between clinical and histological parameters. Ann Transplant 2007;12:30-6.

17. De Gasperi A, Mazza E, Prosperi M. Indocyanine green kinetics to assess liver function: Ready for a clinical dynamic assessment in major liver surgery? World J Hepatol 2016;8:355-67.

18. de Graaf W, Hausler S, Heger M, et al. Transporters involved in the hepatic uptake of $(99 \mathrm{~m}) \mathrm{Tc}-\mathrm{mebrofenin}$ and

Cite this article as: Huang C, Chen Z, Wang T, He X, Chen M, Ju W. A marginal liver graft with hyperbilirubinemia transplanted successfully by ischemia-free liver transplantation. Ann Transl Med 2021;9(5):425. doi: 10.21037/atm-20-6296 indocyanine green. J Hepatol 2011;54:738-45.

19. Gustot T, Fernandez J, Garcia E, et al. Clinical Course of acute-on-chronic liver failure syndrome and effects on prognosis. Hepatology 2015;62:243-52.

20. Martins PN, Buchwald JE, Mergental H, et al. The role of normothermic machine perfusion in liver transplantation. Int J Surg 2020;82S:52-60.

21. Cardini B, Oberhuber R, Fodor M, et al. Clinical Implementation of Prolonged Liver Preservation and Monitoring Through Normothermic Machine Perfusion in Liver Transplantation. Transplantation 2020;104:1917-28.

22. de Meijer VE, Fujiyoshi M, Porte RJ. Ex situ machine perfusion strategies in liver transplantation. J Hepatol 2019;70:203-5.

23. Huang C, Huang S, Tang Y, et al. Prospective, singlecentre, randomised controlled trial to evaluate the efficacy and safety of ischaemia-free liver transplantation (IFLT) in the treatment of end-stage liver disease. BMJ Open 2020;10:e035374.

24. Laing RW, Mergental H, Yap C, et al. Viability testing and transplantation of marginal livers (VITTAL) using normothermic machine perfusion: study protocol for an open-label, non-randomised, prospective, single-arm trial. BMJ Open 2017;7:e017733.

25. Yang J, Yang L, Wu L, et al. Efficacy and Safety of Steroid Therapy for Posttransplant Hyperbilirubinemia Caused by Early Allograft Dysfunction: A Randomized Controlled Trial. Med Sci Monit 2019;25:1936-44.

26. Anwar A, Lee JM. Medical Management of Brain-Dead Organ Donors. Acute Crit Care 2019;34:14-29.

27. Kumar L. Brain death and care of the organ donor. J Anaesthesiol Clin Pharmacol 2016;32:146-52.

28. Totsuka E, Dodson F, Urakami A, et al. Influence of high donor serum sodium levels on early postoperative graft function in human liver transplantation: effect of correction of donor hypernatremia. Liver Transpl Surg 1999;5:421-8.

(English Language Editor: C. Betlazar-Maseh; Quality Control Editor: J. Gray) 